\title{
Collimation of energetic electrons from laser-target interaction by magnetized target-back plasma preformed by a long-pulse laser
}

\author{
H. B. Zhuo, ${ }^{1}$ Z. L. Chen, ${ }^{2}$ Z. M. Sheng,,${ }^{3,4}$ M. Chen,${ }^{3}$ T. Yabuuchi,,${ }^{5}$ M. Tampo,${ }^{5}$ M. \\ Y. Yu, ${ }^{6,7}$ X. H. Yang, ${ }^{1}$ C. T. Zhou, ${ }^{1,8}$ K. A. Tanaka, ${ }^{2,5}$ J. Zhang, ${ }^{3}$ and R. Kodama ${ }^{2,5}$ \\ ${ }^{1}$ College of Science, National University of Defense Technology, Changsha 410073, P. R. China \\ ${ }^{2}$ Graduate School of Engineering, Osaka University 2-1 Yamada-oka, Suita, Osaka 565-0871, Japan \\ ${ }^{3}$ MOE Key Laboratory for Laser Plasmas and Department of Physics and Astronomy, \\ Shanghai Jiao Tong University, Shanghai 200240, P. R. China \\ ${ }^{4}$ Department of Physics, SUPA, University of Strathclyde, Glasgow G4 0NG, UK \\ ${ }^{5}$ Institute of Laser Engineering, Osaka University 2-6 Yamada-oka, Suita, Osaka 565-0871, Japan \\ ${ }^{6}$ Institute for Fusion Theory and Simulation and Department of Physics, \\ Zhejiang University, Hangzhou 310027, P. R. China \\ ${ }^{7}$ Institute for Theoretical Physics I, Ruhr University, Bochum D-44780, Germany \\ ${ }^{8}$ Institute of Applied Physics and Computational Mathematics, Beijing 100094, P. R. China
}

(Dated: April 13, 2014)

\begin{abstract}
It is demonstrated experimentally that the presence of a long-pulse laser created backplasma on the target backside can focus the relativistic electrons produced by short-pulse laser interaction with the front of a solid target. Comparison to that without the backplasma, the number density of the fast electrons is increased by one order of magnitude and their divergence angle is reduced five fold. The effect can be attributed to the absence of the backside sheath electric field and the collimation effect of the megagauss baroclinic magnetic field there. Such a scheme could be of considerable benefit to fast ignition inertial fusion.
\end{abstract}

PACS numbers: $52.25 \mathrm{Fi}, 52.40 . \mathrm{Mj}, 52.27 . \mathrm{Kk}$

Energetic electron (EE) bunches are useful for fast ignition in inertial fusion [1-4], realization of high energy density states [5], compact particle accelerators [7] and novel radiation sources [8], as well as in medical therapy [6]. Fast ignition, for example, demands electron energy deposition at the kilojoule level inside the fuel pellet core [1]. EE bunches produced in intense short-pulse laser-solid target interaction can have fairly high number $\left(10^{14}\right)$, charge $(10 \mu \mathrm{C})$, and energy (several tens joules) $[9,10]$. However, they also have a large divergence angle $\theta_{d}\left(\sim 30^{\circ}-50^{\circ}\right)$ and therefore are hardly applicable. It is thus essential to reduce the spatial spread of the EEs for practical applications.

Collimation of the EEs by the intense magnetic fields induced by their return currents has been proposed [11] and investigated extensively for different target designs $[12,13]$. Using a prepulse to produce an azimuthal magnetic field can also reduce the fast-electron divergence and increase the electron current density $[14,15]$. On the other hand, due to the ubiquitous presence of orthogonal density and temperature gradients, multi-megagauss magnetic fields are easily generated baroclinically in the expanding plasma created by a long-pulse laser interacting with a target [16-18]. The baroclinic magnetic field $(\mathrm{BMF})$ is given by $\partial_{t} \boldsymbol{B}=\nabla T_{e} \times \nabla n_{e} / n_{e} e$, where $t$ is the time, $\boldsymbol{B}$ is the magnetic field, $e, n_{e}$ and $T_{e}$ are electron charge, density and temperature, respectively. The BMF is on a much longer timescale (few hundred ps) than that (few tens fs) of the EEs generated by intense short laser pulses.

In this Letter, we show that in the presence of a magne- tized plasma pre-generated by a long-pulse laser impinging the backside of the target, the intrinsically divergent EEs that have passed through the target can be collimated by the toroidal BMF. The backside plasma also allows the EEs to propagate more stably and suppresses the formation of the target normal sheath electric field there by partially neutralizing the less energetic hot electrons from the target front. As a result, a tight EE bunch with high energy and charge densities can be produced. The proposed process is schematically illustrated and discussed in Fig. 1(a).

To bend the trajectory of a fast electron of speed $v$ at an angle $\theta_{d}$ with respect to the axial $(z)$ direction, the magnetic field should satisfy [14]

$$
B_{\phi} L_{r} \geq \frac{\gamma v m_{e}}{e} S
$$

where $m_{e}$ is electron mass, $L_{r}$ is the scale length of the transverse ( $r$-direction) magnetic field, $\gamma=(1-$ $\left.v^{2} / c^{2}\right)^{-1 / 2}$ is the Lorentz factor, and $S=1-\cos \left(\theta_{d} / 2\right)$. For a fast electron generated at the front side of the target by the main laser pulse of intensity $I_{0}$ and wavelength $\lambda_{0}$, the factor $\gamma v$ is determined by the corresponding ponderomotive force [17]. Accordingly, the condition for deflecting the divergent electron towards the axial direction can be written as [14]

$$
B_{\phi} L_{r} \geq \frac{m_{e} c}{e} \sqrt{1+\frac{I_{0} \lambda_{0}^{2}}{1.38 \times 10^{18} \mathrm{Wcm}^{-2} \mu \mathrm{m}^{2}}} S,
$$

where $c$ is vacuum light speed. 


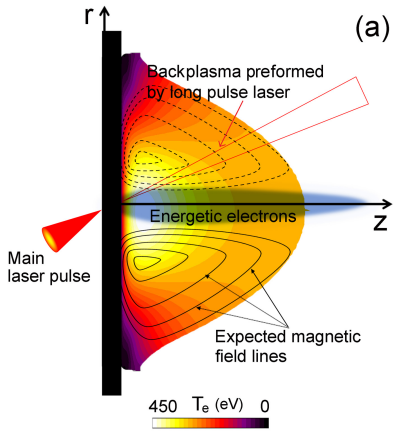

(a)

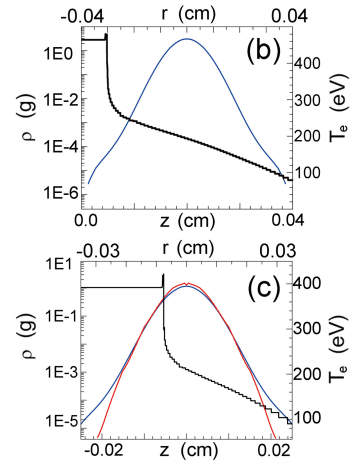

FIG. 1: (color online.) (a) Schematic representation of the target backside plasma. The electron temperature distribution is drawn basing on the results (at 400 ps) from hydrodynamic simulations of the interaction of a long-pulse laser with the backside of a planar $\mathrm{Al}$ target. The contours (thin black closed curves) depict the expected toroidal baroclinic magnetic field, which is peaked around the edge of the laser focal spot and decreases to zero on the axis $(r=0)$ and at large distances from the focal spot. The EEs (blue region) created and accelerated by the targetfront laser are focused into a narrow bunch by the strong magnetic field. The hydrodynamic simulation results for the backplasma electron density (black) along the $z$ axis, and temperature (blue) along the $r$ at the position of $0.01 \mathrm{~cm}$ away from rear surface of the target, are shown in (b) for the $\mathrm{Al}$ target with flat backside, and (c) for two plastic targets with different backside curvatures (blue: infinite, and red: $2.5 \mathrm{~mm}$ ).

We use the radiative hydrodynamics code MULTI2D [20] to simulate the laser-excited plasma expansion and magnetic field generation at the target backside. In accordance with the experimental parameters, a $2 \mathrm{~J} p$ polarized $1.053 \mu \mathrm{m} 400 \mathrm{ps}$ long laser pulse is focused on a $50 \mu \mathrm{m}$-thick Al target with a $\sim 300 \mu \mathrm{m}$ spot diameter. Fig. 1(b) shows that the electron density gradient is mainly in the axial direction, as expected. Thus, the axial temperature gradient does not contribute to the BMF generation. The magnitude of the BMF is then roughly given by $B_{0} \sim T_{e} t / l_{n} l_{T}$, where $l_{n}$ and $l_{T}$ are the scalelengths of the density and temperature, respectively. For $T_{e} \sim 400 \mathrm{eV}, l_{n} \sim 10 \mu \mathrm{m}$, and $l_{T} \sim 150 \mu \mathrm{m}$ (estimated from Fig. 1(b)), we find that the magnetic field attains $1 \mathrm{MG}$ in $400 \mathrm{ps}$. For $200 \mu \mathrm{m}$-thick plastic targets with different targetback curvatures, our simulations show almost no difference in the axial profiles of the plasma density. On the other hand, the radial temperature gradient increases with the curvature since the plasma is transversely better confined, as shown in Fig. 1(c). Accordingly, with a BMF $\sim 1 \mathrm{MG}$ in the backplasma of $L_{r} \sim 150 \mu \mathrm{m}$, the collimation condition (2) is readily satisfied for EEs driven by a $I_{0} \lambda_{0}^{2}=5 \times 10^{18}$ $\mathrm{Wcm}^{-2} \mu \mathrm{m}^{2}$ main laser pulse and exiting the target back with $\theta_{d} \sim 50^{\circ}$.

Collimation of the EEs in the intense laser interaction

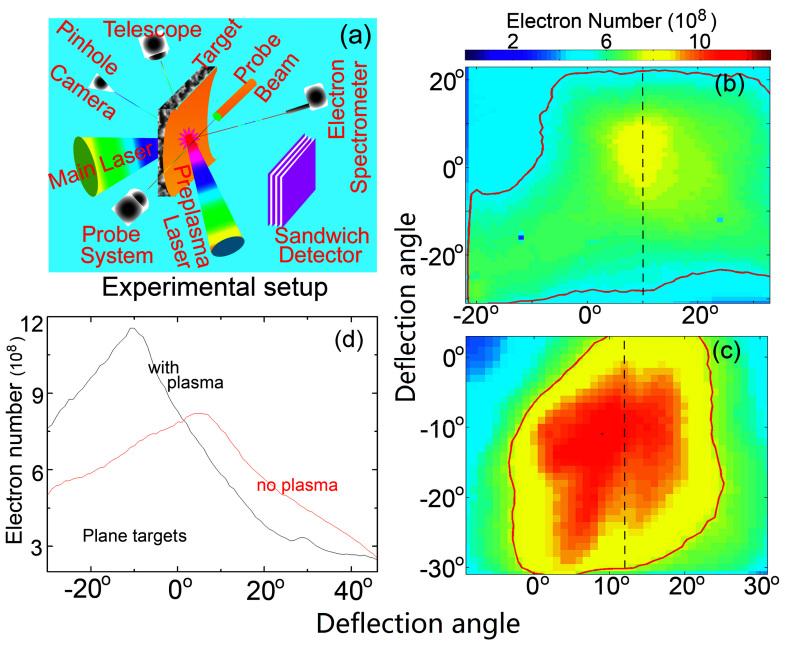

FIG. 2: (Color online.) Experimental setup (a). Observed angular distribution of electrons over $0.4 \mathrm{MeV}$ for planar $\mathrm{Al}$ target with (b) and without (c) preformed plasma on the backside. The $p$-polarized main laser pulse defining the angular direction $\left(0^{\circ}, 0^{\circ}\right)$ is incident obliquely at a $20^{\circ}$ angle on the front surface of the target (such that the target normal is in the $\left(20^{\circ}, 0^{\circ}\right)$ direction. (d) Number of $>0.4 \mathrm{MeV}$ electrons along the grey dashed lines in the panels (b) and (c).

at the target front are tested for both planar Al target$\mathrm{s}$ and plastic targets with concave back surfaces on the GEKKO Module II laser system at the Institute of Laser Engineering, Osaka University. The experimental setup is shown in Fig. 2(a). After a 400 ps time delay for preplasma formation at the back surface by a long-pulse laser, EEs are generated at the flat target front surface at an $20^{\circ}$ incident angle by a $p$-polarized $10 \mathrm{~J} 1.053 \mu \mathrm{m}$ $0.6 \mathrm{ps}$ short-pulse laser. The laser pulse is focused by an $\mathrm{f} / 3.8$ off-axis parabolic mirror into a $20 \mu \mathrm{m}$ diameter spot with peak intensity $\sim 5 \times 10^{18} \mathrm{Wcm}^{-2}$. To probe the energy-resolved angular distribution of the EEs along the axis of the main laser, a sandwich detector is placed $\sim 4 \mathrm{~cm}$ away from the targetback. It consists of four layers of photo-stimulated-luminescence (PSL) imaging plates (IPs) with in-between copper filters for detecting $0.4 \mathrm{MeV}, 3 \mathrm{MeV}, 6 \mathrm{MeV}$, and $10 \mathrm{MeV}$ electrons. A radiochromic film (RCF) layer and a CR-39 layer in front of the IPs are used to monitor the angular distribution of energetic protons. A $10 \mu \mathrm{m}$ thick $\mathrm{Al}$ foil is placed in front of the detectors to shield them from the target debris. The entire detector system is wrapped with black tapes and Al foils to block stray light. Tests showed that the small number of protons from target normal sheath acceleration can be completely stopped from arriving at the IPs by the RCF and CR-39 layers.

The angular distribution of the EEs behind a plain $\mathrm{Al}$ target without a preformed backplasma is shown in Fig. 2 (b). We see that the electrons are of low density $\left(8 \times 10^{8}\right)$ and have a rather irregular average angular spread of 


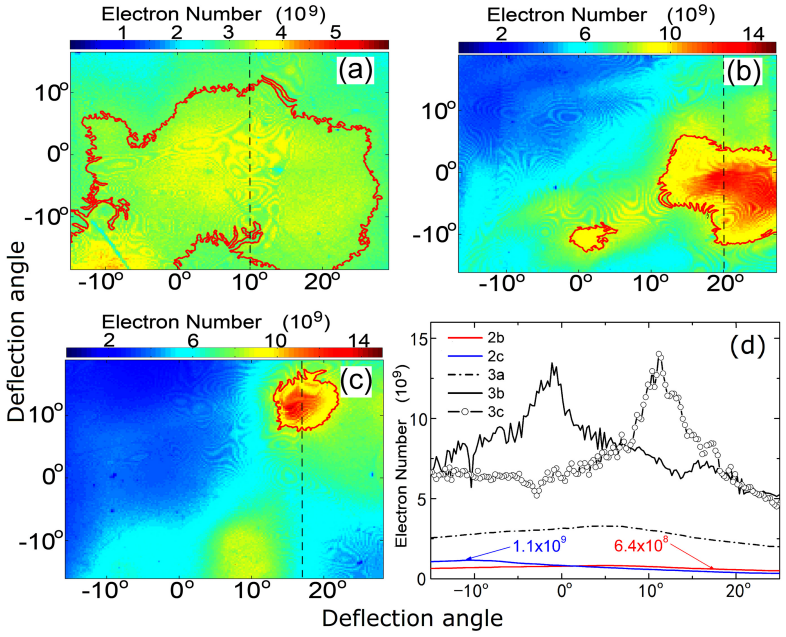

FIG. 3: (Color online.) Experimental angular distributions of energetic electrons over $0.4 \mathrm{MeV}$ for different cylindrical target back curvatures: (a) infinite (or planar), (b) $5.0 \mathrm{~mm}$, and (c) $2.5 \mathrm{~mm}$, for $200 \mu \mathrm{m} \times\left(5 \times 5 \mathrm{~mm}^{2}\right)$ plastic targets. The curvature axes in (b) and (c) are perpendicular and parallel, respectively, to the laser polarization. (d) Electron number (obtained from the sandwich detector using photo stimulated luminescence) along the dashed lines in Figs. 2(b), 2(c), 3(a), $3(\mathrm{~b})$, and $3(\mathrm{c})$, denoted by $2 \mathrm{~b}, 2 \mathrm{c}, 3 \mathrm{a}, 3 \mathrm{~b}$, and $3 \mathrm{c}$, respectively.

more than $50^{\circ}$ around the laser axis at $0^{\circ}$. Fig. $2(\mathrm{c})$ shows that, in the presence of a performed plasma behind the target, the electrons are much more uniform and of much higher density $\left(1.2 \times 10^{9}\right)$. They are also much better collimated, with the average angular spread reduced to $\sim 30^{\circ}$. It should be mentioned that despite their near axial direction and narrow spread, the mean direction of the EEs varies somewhat from shot to shot, which can be attributed to uncertainties in the configuration (which determines the focusing direction) of the self-generated magnetic field.

In order to see in more detail the effect of the backplasma and the self-generated magnetic field, we have also considered plastic targets with different concave back surfaces. As discussed, the latter determines the backplasma density and temperature as well as their gradients, and therefore also the self-generated BMF. The results are shown in Fig. 3. One can see that as the curvature radius is reduced from infinite (a) to $5 \mathrm{~mm}$ (b) to $2.5 \mathrm{~m}$ $\mathrm{m}(\mathrm{c})$, the spatial spread of the EEs becomes smaller and their number density higher. Similar to that for the flat $\mathrm{Al}$ target, the deviation of the EEs from the exact axial direction of the main laser is due to uncertainty in the configuration of the baroclinic magnetic field.

For comparison, Fig. 3(d) shows the electron number along the dashed lines for the five cases in Figs. 2(b) and $2(\mathrm{c})$ and $3(\mathrm{a})-3(\mathrm{c})$. We see that the electrons behind the curved target backs are much better collimated. Their number is also more than one order of magnitude higher than that behind the flatback targets. These results

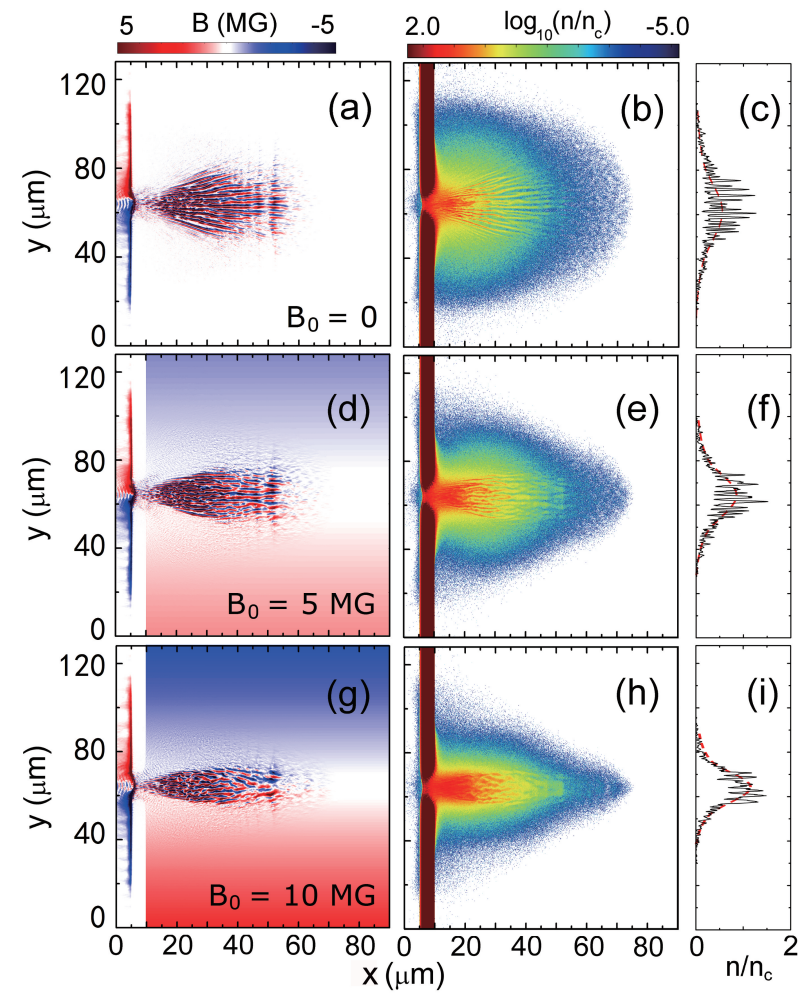

FIG. 4: (Color online.) Results from 2D PIC simulations at $t=80$ laser periods from the laser impact: magnetic field distribution (left column), distribution of $>1 \mathrm{MV}$ energetic electrons (center column), and electron density at $x=30$ $\mu \mathrm{m}$ (right column), for $B_{0}=0 \mathrm{MG}$ (top row), $B_{0}=5 \mathrm{MG}$ (center row), and $B_{0}=10 \mathrm{MG}$ (bottom row). The $5 \mu \mathrm{m}-$ thick solid-density $\left(20 n_{c}\right.$, where $\left.n_{c}=1.1 \times 10^{21} / \mathrm{cm}^{3}\right)$ target is at $5<x[\mu \mathrm{m}]<10$. Note that the magnetic fields associated with the laser-target interaction and energetic-electron filamentation are relatively much stronger and localized than the prescribed megagauss model baroclinic magnetic fields, appearing as large blue and red shaded regions in (d) and (g).

represent a significant improvement over that of the exiting experiments [20]. In fact, Figs. 3(a) and 3(c) show that the divergence of the EEs is reduced from about $\theta_{d} \sim 30^{\circ}$ for the flatback target to about $\theta_{d} \sim 10^{\circ}$ for the curveback targets. Moreover, the number density in the center region is increased fivefold. We note that if all the EEs in the original $30^{\circ}$ divergence angle were focused into the $10^{\circ}$ divergence angle, the density enhancement factor would be $\sim 9.5$, which is larger than the experimental value $\sim 5.0$. The discrepancy can be attributed to filamentation $[22,23]$ of the fast EEs as they propagate in the underdense region of the backplasma, since some electrons are scattered away from the EE bunch, as can be seen in Figs. 3(b) and 3(c).

To further clarify the collimation effect of the BMF as observed in our experiments, we have carried out 2D simulations of the process using the particle-in-cell (PIC) simulation code PDLPICC2D [7]. A $3.425 \times 10^{19} \mathrm{~W} / \mathrm{cm}^{2}$ 
laser pulse of 100 laser cycles in FWHM (full width at half maximum) is focused to a $10 \lambda_{0}$-diameter spot on a $5 \mu \mathrm{m}$-thick solid density $\left(n_{0}=20 n_{c}\right)$ plasma slab at $x=$ $5 \mu \mathrm{m}$. The preformed backplasma is modeled by an exponentially decaying plasma, with density $n_{0} \exp [-(x-$ 10) $/ 10] \mathrm{cm}^{-3}$, attached to the back of the target. The model BMF is given by $\boldsymbol{B}=2 B_{0}\left(y-0.5 L_{y}\right) \boldsymbol{e}_{z} / L_{y}$, where $L_{y}=128 \mu \mathrm{m}$ is the height of the simulation box. Fig. 4 shows the distributions of the magnetic fields (left column) and $>1 \mathrm{MeV}$ EEs (center and right columns) for $B_{0}=0,5$, and $10 \mathrm{MG}$, corresponding to: the collimation condition (2) not satisfied, satisfied, and well satisfied, respectively. Note that the magnetic fields associated with the front laser and the filamentation of the EEs are much stronger and much more localized than the model BMF (appearing only as red and blue colored shades in Figs. $4(\mathrm{~d})$ and $4(\mathrm{~g}))$ behind the target. However, the former fields are not directly of our interest here.

In Fig. 4 we can see that filamentation of the EEs occurs as they exit the rear side of the target, and in the absence of the BMF the filaments, together with their induced magnetic fields, are highly divergent. As $B_{0}$ increases, the electron filaments, or the electron jets as a whole, become better collimated and focused, even though they appear to be locally unstable to kink or sausage type instabilities and tend to breakup and then coalesce the originally thin filaments, as can be seen in Fig. 4, right column, for the line profiles of the EE density at $x=30$ $\mu \mathrm{m}$. We can also see that, because of improved focusing, the peak EE number density is enhanced by a factor of about 4 as $B_{0}$ increases from 0 to 10 MG. Except for the precise location (unpredictable in the experiments) of the peaks, these simulation results agree qualitatively well with that (Fig. 3(d)) from the experiments.

In summary, focusing of relativistic laser generated EEs from the front of a solid target by the BMF in a backplasma created at the target backside by a long pulse laser is demonstrated experimentally and by PIC simulations. The EEs, originally with large divergence angle $\left(\theta_{d} \sim 50^{\circ}\right)$, are collimated into a tight bunch with $\theta_{d} \sim 10^{\circ}$ as they propagate in the backplasma. The focusing and collimation are shown be effected by the strong BMF generated by the nonparallel density and temperature gradients in the backplasma. The estimated condition for collimation and the results from the PIC simulations for the process agree reasonably well with that from our experiments. The proposed scheme provides a simple and effective way for collimating a large number of initially highly divergent EEs, such that an electron bunch of high energy and charge densities can be produced.

This work is supported by the National Research Program of China (2013CBA01504), the National Natural Science Foundation of China (11175253, 91230205, 11275269, 11205194, 11247007, and 11374262, 11220101002), the Research Program of NUDT, ITER-
CN (2013GB104004), and the Open Fund of the State Key Laboratory of High Field Laser Physics at SIOM. We would like to thank the Tianjin National Supercomputing Center for providing their computing facilities.

[1] M. Tabak, J. Hammer, M. E. Glinsky, W. L. Kruer, S. C. Wilks, J. Woodworth, E. M. Campbell, M. D. Perry, and R. J. Mason, Phys. Plasmas 1, 1626 (1994).

[2] M. Tabak, D. S. Clark, S. P. Hatchett, M. H. Key, B. F. Lasinski, R. A. Snavely, S. C. Wilks, R. P. J. Town, R. Stephens, E. M. Campbell, R. Kodama, K. Mima, K. A. Tanaka, S. Atzeni, and R. Freeman, Phys. Plasmas 12, 057305 (1994).

[3] R. Kodama, P. A. Norreys, K. Mima, A. E. Dangor, R. G. Evans, H. Fujita, Y. Kitagawa, K. Krushelnick, T. Miyakoshi, N. Miyanaga, T. Norimatsu, S. J. Rose, T. Shozaki, K. Shigemori, A. Sunahara, M. Tampo, K. A. Tanaka, Y. Toyama, T. Yamanaka, and M. Zepf, Nature 412, 798 (2001).

[4] R. Kodama, H. Shiraga, K. Shigemori, Y. Toyama, S. Fujioka, H. Azechi, H. Fujita, H. Habara, T. Hall, Y. Izawa, T. Jitsuno, Y. Kitagawa, K. M. Krushelnick, K. L. Lancaster, K. Mima, K. Nagai, M. Nakai, H. Nishimura, T. Norimatsu, P. A. Norreys, S. Sakabe, K. A. Tanaka, A. Youssef, M. Zepf, and T. Yamanaka, Nature 418, 933 (2002).

[5] R. Kodama, Y. Sentoku, Z. L. Chen, G. R. Kumar, S. P. Hatchett, Y. Toyama, T. E. Cowan, R. R Freeman, J. Fuchs, Y. Izawa, M. H. Key, Y. Kitagawa, K. Kondo, T. Matsuoka, H. Nakamura, M. Nakatsutsumi, P. A. Norreys, T. Norimatsu, R. A. Snavely, R. B. Stephens, M. Tampo, K. A. Tanaka, and T. Yabuuchi, Nature 432 , 1005 (2004).

[6] S.V Bulanov, T.Zh Esirkepov, V.S Khoroshkov, A.V Kuznetsov, and F. Pegoraro, Phys. Lett. A 299, 240 (2002).

[7] H. B. Zhuo, Z. L. Chen, W. Yu, Z. M. Sheng, M. Y. Yu, Z. Jin, and R. Kodama, Phys. Rev. Lett. 105, 065003 (2010).

[8] Z. Jin, Z. L. Chen, H. B. Zhuo, A. Kon, M. Nakatsutsumi, H. B. Wang, B. H. Zhang, Y. Q. Gu, Y. C. Wu, B. Zhu, L. Wang, M. Y. Yu, Z. M. Sheng, and R. Kodama, Phys. Rev. Lett. 107, 265003 (2011).

[9] J. S. Green, V. M. Ovchinnikov, R. G. Evans, K. U. Akli, H. Azechi, F. N. Beg, C. Bellei, R. R. Freeman, H. Habara, R. Heathcote, M. H. Key, J. A. King, K. L. Lancaster, N. C. Lopes, T. Ma, A. J. MacKinnon, K. Markey, A. McPhee, Z. Najmudin, P. Nilson, R. Onofrei, R. Stephens, K. Takeda, K. A. Tanaka, W. Theobald, T. Tanimoto, J. Waugh, L. Van Woerkom, N. C. Woolsey, M. Zepf, J. R. Davies, and P. A. Norreys, Phys. Rev. Lett. 100, 015003 (2008).

[10] K. L. Lancaster, J. S. Green, D. S. Hey, K. U. Akli, J. R. Davies, R. J. Clarke, R. R. Freeman, H. Habara, M. H. Key, R. Kodama, K. Krushelnick, C. D. Murphy, M. Nakatsutsumi, P. Simpson, R. Stephens, C. Stoeckl, T. Yabuuchi, M. Zepf, and P. A. Norreys, Phys. Rev. lett 98, 125002 (2007)

[11] A. R. Bell and R. J. Kingham, Phys. Rev. Lett. 91 035003 (2003). 
[12] A. P. L. Robinson and M. Sherlock, Phys. Plasmas 14, 083105 (2007).

[13] S. Kar, A. P. L. Robinson, D. C Carroll, O Lundh, K. Markey, P. McKenna, P. Norreys, and M. Zepf, Phys. Rev. Lett. 102, 055001 (2009).

[14] A. P. L. Robinson, M. Sherlock and P. A. Norreys, Phys. Rev. Lett. 100, 025002 (2008).

[15] R. H. H. Scott, C. Beaucourt, H. P. Schlenvoigt, K. Markey, K. L. Lancaster, C. P. Ridgers, C. M. Brenner, J. Pasley, R. J. Gray, I. O. Musgrave, A. P. L Robinson, K. Li, M. M. Notley, J. R. Davies, S. D. Baton, J. J. Santos, J. L. Feugeas, P. Nicolai, G. Malka, V. T. Tikhonchuk, P. McKenna, D. Neely, S. J. Rose, and P. A. Norreys, Phys. Rev. Lett. 109, 015001 (2012).

[16] J. A. Stamper, K. Papadopoulos, R. N. Sudan, S. O. Dean, and E. A. McLean, Phys. Rev. Lett. 26, 1012 (1971).

[17] O. Willi, P. T. Rumsby, and C. Duncan, Opt. Commun. 37, 40 (1981).

[18] M. Borghesi, A. J. MacKinnon, A. R. Bell, R. Gaillard, and O. Willi, Phys. Rev. Lett. 81, 112 (1998).

[19] R. Remis, R. Schmaltz and J. Meyer-Ter-Vehn, Comp. Phys. Comm. 49, 475 (1988).

[20] T. Yabuuchi, K. Adumi, H. Habara, R. Kodama, K. Kondo, T. Tanimoto, K. A. Tanaka, Y. Sentoku, T. Matsuoka, Z. L. Chen, M. Tampo, A. L. Lei and K. Mima, Phys. Plasmas 14, 040706 (2007).

[21] S. C. Wilks and W. L. Kruer, IEEE J. Quantum Electron 33, 1954 (1997).

[22] M. Tatarakis, F. N. Beg, E. L. Clark, A. E. Dangor, R. D. Edwards, R. G. Evans, T. J. Goldsack, K. W. D. Ledingham, P. A. Norreys, M. A. Sinclair, M. S. Wei, M. Zepf, and K. Krushelnick, Phys. Rev. Lett. 90, 175001 (2003).

[23] M. S. Wei, F. N. Beg, E. L. Clark, A. E. Dangor, R. G. Evans, A. Gopal, K. W. D. Ledingham, P. McKenna, P. A. Norreys, M. Tatarakis, M. Zepf, and K. Krushelnick, Phys. Rev. E 70, 056412 (2004). 